Erratum

\title{
Erratum to "Intratumoral Heterogeneity of MAGE-C1/CT7 and MAGE-C2/CT10 Expression in Mucosal Melanoma"
}

\author{
A. Curioni-Fontecedro ${ }^{D},{ }^{1}$ R. Pitocco, ${ }^{2}$ N. L. Schoenewolf, ${ }^{3}$ D. Holzmann, ${ }^{4}$ D. Soldini, ${ }^{5}$ \\ R. Dummer, ${ }^{3}$ S. Calvieri, ${ }^{2}$ H. Moch, ${ }^{5}$ A. Fitsche, ${ }^{5}$ and D. Mihic-Probst $\oplus^{5}$ \\ ${ }^{1}$ Department of Oncology, University Hospital Zurich, 8091 Zurich, Switzerland \\ ${ }^{2}$ Department of Dermatology, Policlinico Umberto I, University of Rome La Sapienza, 00185 Rome, Italy \\ ${ }^{3}$ Department of Dermatology, University Hospital Zurich, 8091 Zurich, Switzerland \\ ${ }^{4}$ Department of Otorhinolaryngology, University Hospital Zurich, 8091 Zurich, Switzerland \\ ${ }^{5}$ Institute of Surgical Pathology, University Hospital Zurich, 8091 Zurich, Switzerland
}

Correspondence should be addressed to A. Curioni-Fontecedro; alessandra.curioni@usz.ch

Received 17 June 2019; Accepted 18 June 2019; Published 22 July 2019

Copyright (C) 2019 A. Curioni-Fontecedro et al. This is an open access article distributed under the Creative Commons Attribution License, which permits unrestricted use, distribution, and reproduction in any medium, provided the original work is properly cited.

In the article titled "Intratumoral Heterogeneity of MAGEC1/CT7 and MAGE-C2/CT10 Expression in Mucosal Melanoma" [1], there was an error in the authors' order, as Dr. D. Mihic-Probst should be listed as the last author. The corrected authors' list and affiliations' list are shown above.

\section{References}

[1] A. Curioni-Fontecedro, R. Pitocco, N. L. Schoenewolf et al., "Intratumoral heterogeneity of MAGE-C1/CT7 and MAGEC2/CT10 expression in mucosal melanoma," BioMed Research International, vol. 2015, Article ID 432479, 5 pages, 2015. 


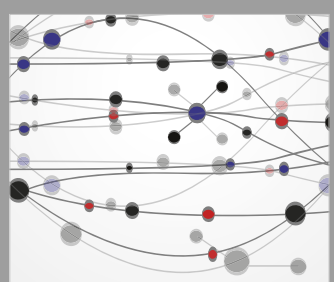

The Scientific World Journal
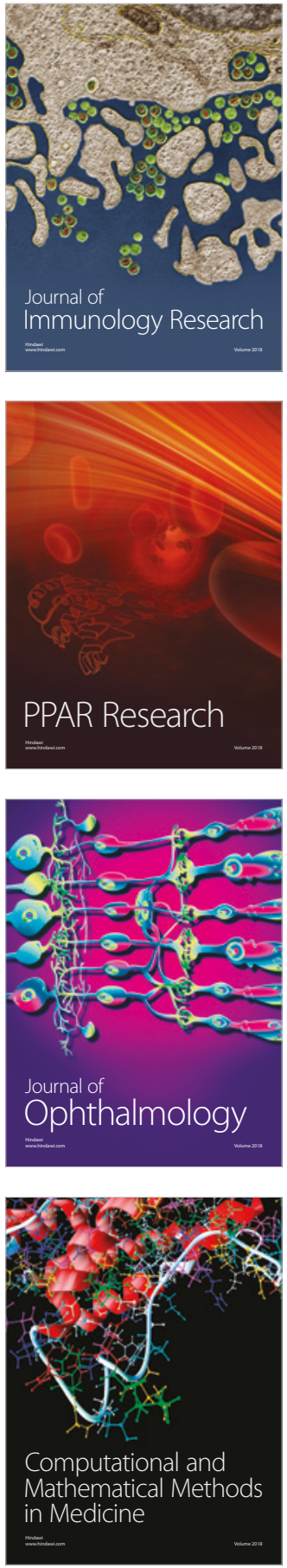



Gastroenterology Research and Practice



\section{Hindawi}

Submit your manuscripts at

www.hindawi.com
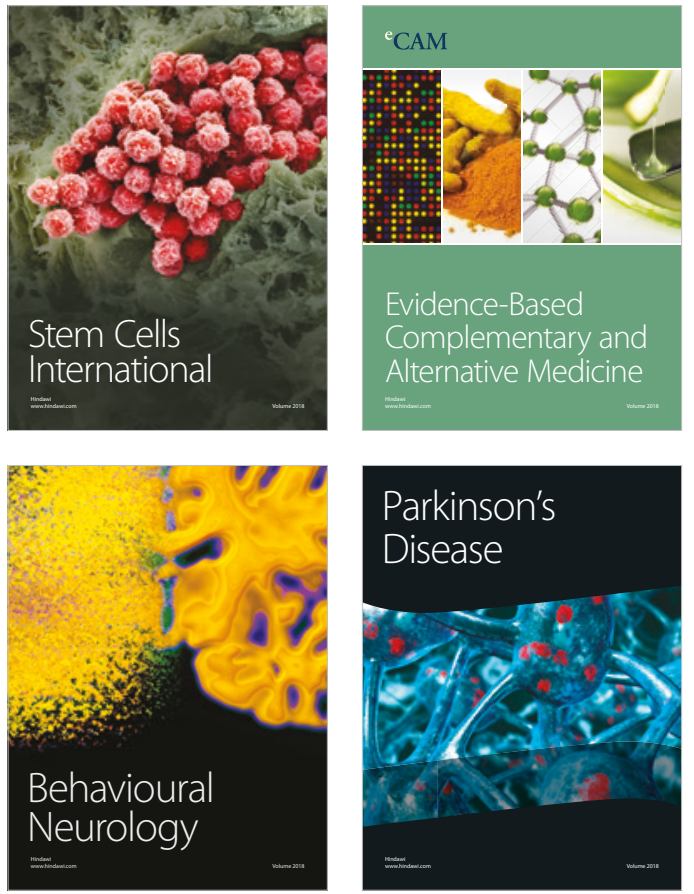

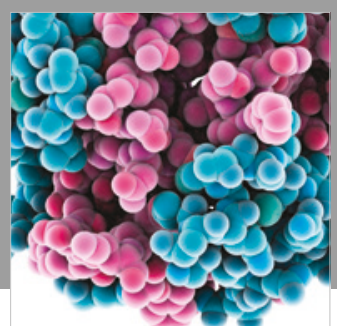

ournal of

Diabetes Research

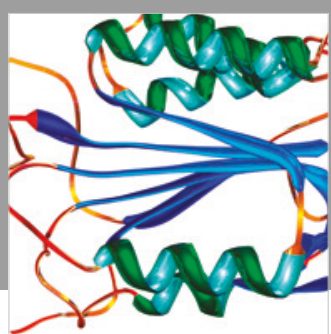

Disease Markers
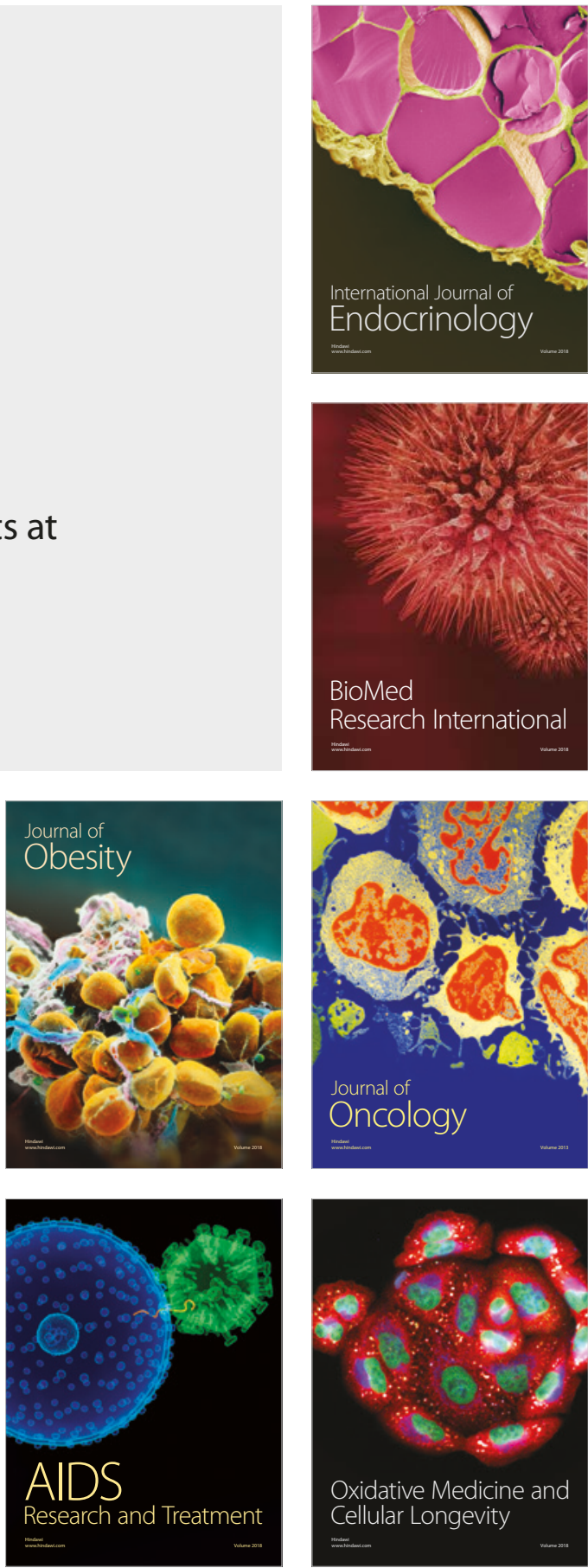